\title{
Recontacting or not recontacting? A survey of current practices in clinical genetics centres in Europe
}

\author{
Fabio Sirchia ${ }^{1}$ - Daniele Carrieri $\mathbb{D}^{2}$ - Sandi Dheensa ${ }^{3}$ - Caroline Benjamin ${ }^{4,5} \cdot$ Hülya Kayserili ${ }^{6}$. \\ Christophe Cordier $\mathbb{B}^{7} \cdot$ Carla G. van $\mathrm{El}^{8}$ - Peter D. Turnpenny ${ }^{9} \cdot$ Bela Melegh ${ }^{10}$ - Álvaro Mendes ${ }^{11}$. \\ Tanya F. Halbersma-Konings ${ }^{12} \cdot$ Irene M. van Langen $^{12} \cdot$ Anneke M. Lucassen $^{3,13}$ - Angus J. Clarke $\mathbb{B}^{14}$. \\ Francesca Forzano ${ }^{15}$. Susan E. Kelly ${ }^{2}$
}

Received: 17 November 2017 / Revised: 16 February 2018 / Accepted: 23 February 2018 / Published online: 23 April 2018

(c) European Society of Human Genetics 2018

\begin{abstract}
Advances in genomic medicine are improving diagnosis and treatment of some health conditions, and the question of whether former patients should be recontacted is therefore timely. The issue of recontacting is becoming more important with increased integration of genomics in 'mainstream' medicine. Empirical evidence is needed to advance the discussion over whether and how recontacting should be implemented. We administered a web-based survey to genetic services in European countries to collect information about existing infrastructures and practices relevant to recontacting patients. The majority of the centres stated they had recontacted patients to update them about new significant information; however, there were no standardised practices or systems in place. There was also a multiplicity of understandings of the term 'recontacting', which respondents conflated with routine follow-up programmes, or even with post-test counselling. Participants thought that recontacting systems should be implemented to provide the best service to the patients and families. Nevertheless, many barriers to implementation were mentioned. These included: lack of resources and infrastructure, concerns about potential negative psychological consequences of recontacting, unclear operational definitions of recontacting, policies that prevent healthcare professionals from recontacting, and difficulties in locating patients after their last contact. These barriers are also intensified by the highly variable development (and establishment) of the specialties of medical genetics and genetic counselling across different European countries. Future recommendations about recontacting need to consider these barriers. It is also important to reach an 'operational definition' that can be useful in different countries.
\end{abstract}

\section{Introduction}

The question about whether healthcare professionals (HCPs) should recontact patients if new genetic information becomes available is becoming more pressing as large-scale genomic collections facilitate new interpretations more

These authors contributed equally: Fabio Sirchia, Daniele Carrieri, Francesca Forzano, Susan E. Kelly.

Electronic supplementary material The online version of this article (https://doi.org/10.1038/s41431-018-0131-5) contains supplementary material, which is available to authorized users.

Daniele Carrieri

d.carrieri@exeter.ac.uk

Extended author information available on the last page of the article frequently [1-4]. A systematic review on recontacting conducted by Otten et al. [5] highlighted that the question about recontacting has become more important after the introduction of next-generation sequencing, and the concomitant increase in variants of unknown significance (VUSs). Otten et al. define recontacting as the HCPs' potential 'ethical and/or legal obligation to recontact former patients in light of new genetic information'.

The use of the word 'former' implies that patients have previously been discharged from care, but we acknowledge that the question about recontacting can arise even without a formal discharge (depending on the service, e.g. general practitioners tend not to discharge patients) if a long period of time has elapsed.

The literature tends to view recontacting as ethically desirable even if currently it is not the standard of care. Nevertheless, there are arguments both in favour and 
against recontacting. On one hand, recontacting is seen as potentially benefiting the health and psychological wellbeing of patients and their relatives. However there are also concerns about the psychosocial complexity of recontacting [6], including the concern that it may be experienced by patients as an intrusion of privacy, a breach of their right not to know [7], as a cause of anxiety and stress, also any impact in relation to health and life insurance $[1,8,9]$. Practical issues such as lack of time, dedicated staff, resources and infrastructure to reliably track patients are also often mentioned as important barriers to recontacting [10-13].

A survey of regulations and practices of genetic counselling in 38 European countries highlighted that recontacting is one of the least covered topics in both professional guidelines and national policies [14]. To date, there is only one set of professional guidelines specifically addressing recontacting and these are from the USA [2]. This holds the primary care physician responsible for alerting patients about the potential need for recontacting because genetic services often do not maintain an ongoing relationship with the patients. The 2008 revision [15] of this guideline recommends that any clinical genetic laboratory scientist who becomes aware of new information should make an effort to alert the referring physician if the clinical interpretation of a genetic finding changes. However, this may not always be an easy task for the laboratory, due to lack of time and resources. The problem is further complicated by the fact that-as genetic testing is increasingly offered by specialties other than clinical genetics (e.g. cardiology, oncology, neurology), in so-called mainstream medicine [16] - the referring physician is sometimes difficult to identify.

HCPs and patients interviewed in the UK both expressed concerns about the feasibility of routine recontacting within current resource constraints, but also about a lack of clarity over roles and responsibilities [4, 6]. Some authors have suggested that patients could take an active part by requesting updates from their clinicians $[17,18]$. However, the idea of sharing responsibility with patients could amplify existing health and social inequalities. For example, more educated, articulate and assertive patients might seek updates from HCPs much more often than less educated or assertive patients, whose needs might be greater [19]. Besides, 'autonomy' is one of the tenets in genetic counselling, and we have to consider that patients can autonomously choose to not seek updates.

More empirical evidence is needed to advance the discussion over whether and how recontacting should be implemented; however, such evidence is still sparse. Carrieri et al. [20] surveyed clinical genetic centres in the UK regarding their recontacting practices and attitudes. Twenty of 24 UK centres responded to this survey. The majority indicated that recontacting patients and family members was appropriate if there was some clinically relevant new information. However, the survey highlighted that there are no standard recontacting practices in the UK and most centres were unsure whether systematic recontacting should be implemented. Many expressed concerns about such implementation, for example fear of being viewed as negligent if they failed to recontact. To provide more empirical data on current recontacting practices, we administered the UK survey to genetic centres in Europe.

\section{Method}

The main objective of the survey was to ascertain whether and how recontacting occurs in clinical genetic centres in Europe. We chose to use the same questions as in the UK survey [20] to combine the data gathered in the UK with the other European countries. The survey was administered between August 2016 and April 2017. A link to the survey was circulated via an e-mail invitation to the President or the contact person of the National Human Genetic Societies listed on the ESHG webpage: https://www.eshg.org/76.0. html. In some countries, the National Human Genetic Societies circulated the survey to their genetic centres. In other countries, the National Human Genetic Societies provided a list of the genetic centres or contact persons and we circulated the survey directly to the named clinical genetic centres referred. For several countries it was impossible to find or to construct a complete list or the number of the clinical genetic centres. We also asked the presidents of the National Societies about the number of centres in their own country, but for most countries we did not receive a clear answer (some of these societies were in the process of undertaking a 'census' of clinical genetics centres in their country). For instance, in some countries, genetic counselling can be performed by biologists or physicians whose main occupation is to work in a small laboratory and who only rarely perform patient counselling. Moreover, in some countries the residency programme in medical genetics is still not developed and there is a lack of medical geneticists and genetic counsellors. The professional role of respondents were diverse, and included Head of the service, genetic counsellor, consultant, and trainee. However, we wanted respondents to complete the survey on behalf of their whole service. To encourage this, we asked respondents (both in the introduction to the survey and in the accompanying email) to discuss the survey questions with their colleagues before filling it in. The survey included closed and open questions with expandable text boxes to elicit explanatory comments and examples. This combination of closed and open questions allowed the research 


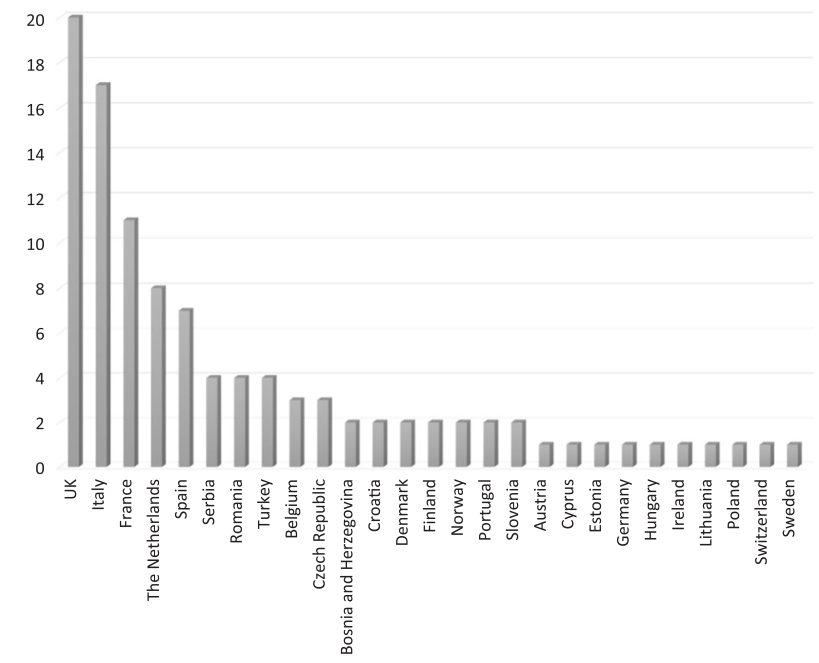

Fig. 1 Number of clinical genetics centres that participated in the survey (grouped by country)

team to collect both quantitative and qualitative data. Freetext responses from the survey were analysed using content analysis [21]—using the survey questions as a framework to categorise the answers. Quantitative closed questions were analysed using descriptive statistics. The data was initially analysed by FS and DC. The analysis was further developed involving the other authors via face-to-face meetings (organised by the ESHG PPPC between 2016 and 2017) and several iterations of comments on drafts of the paper. The survey is provided as Supplementary Information.

\section{Results}

Among the 105 genetic centres that answered the survey (Figure 1), 100 stated that they had recontacted patients or families to update them about new significant information. See Table 1 for a summary of the reasons given. However, only 37 affirmed that they recontact routinely, and 63 indicated that they recontact on an occasional basis.

Among the five genetic centres that stated they had not recontacted patients, three indicated that this was due to insufficient staff and/or resources; one reported that the policy in their country does not allow a direct recontacting of the patients; and one indicated that this was due to the absence of recontacting procedures.

It is worth noting that some centres did not differentiate routine follow-up (e.g. discussing at subsequent and more appropriate times the complications of a patient's condition, or periodical pre-arranged appointments) from recontacting a previously discharged patient in light of new information. Some respondents referred to recontacting as being a process that can also be initiated by the patient (e.g. when new information comes from the proband or his/her family) or
Table 1 Most common reasons for recontacting patients (and potentially relevant family members)

\begin{tabular}{lc}
\hline & $N$ \\
\hline Availability of new test/technologies (test to offer to the patient) & 55 \\
New clinical guidance & 33 \\
Reclassification of VUS & 26 \\
New results (from tests already performed) & 17 \\
Research/clinical trials & 15 \\
Follow-up (continuity of care) & 7 \\
New information coming from relatives of the proband & 5 \\
Propose genetic tests to relatives of the proband & 5 \\
Invite patients to support groups or clinical meetings related to & 4 \\
their health condition & \\
Correction of previous analytical errors & 4 \\
\hline
\end{tabular}

support groups (e.g. when the patient is invited to clinical meetings or support group meetings). In some cases, the responses of one centre combined more than one of these understandings of the term recontacting.

\section{Situations in which recontacting is considered good clinical practice}

The answers to the question about the type of information the genetic centres considered relevant enough to trigger a recontact were coherent with the reasons given for having recontacted patients (summarised in Table 1). Recontacting was considered important, and patients tended to be recontacted, in the light of new, clinically relevant information:

'We consider it relevant to trigger recontact when there is availability of new treatment strategies/ options, (including new phase 2-3 trials), new testing strategies, new information that may have impact on family planning and reproductive options and any new information that may have impact on follow-up of a patients (e.g. cancer susceptibility)'

\section{Recontacting procedures}

Among the 100 centres that responded that they recontacted patients, 41 stated they had developed recontacting procedures, and 59 stated they had not developed them. Within both groups, there were centres that affirmed they recontacted routinely and occasionally.

The procedures reported varied across and within countries and the majority of the centres used more than one method. The procedures are summarised in Table 2, and some examples are provided with quotations below: 
'Patients attended in our hospital have signed an IC [informed consent] accepting or refusing to be recontacted and we follow their decisions'

'We contact the patient and mention the reason for the call briefly. If they do not accept, we mark the patient file accordingly. We ask them if they do not want to be contacted by our institution. If the answer is no, we again mark it on the file as 'do not call the patient again",

\section{Use of databases for recontacting purposes}

Eighty-four centres stated that they used a database for recontacting purposes. Among these, 56 indicated that they use databases for both demographics (to access patient's contact details) and clinical genetic purposes (e.g., link the patient with other relatives' records, access laboratory results, access and review medical notes, flag/unflag patients). Twenty-three reported their use of databases only for demographic purposes. Five centres did not specify in which ways they use databases for recontacting.

The way databases were used for recontacting appeared to vary considerably across and within countries. This seemed to be mostly the result of the different programs/ software packages used.

Furthermore, respondents' answers suggested that most of the existing databases were not easily usable for recontacting purposes, e.g. they did not allow the recording of patient recontacting preferences (which appeared to be recorded instead on paper in the patient file).

'We use databases to find patients with the relevant condition and get their contact details; occasionally we flag them for future recontact but this is limited currently, though we may expand it when our new database is working (is currently being implemented)'

'Not one database in use contains all information and is suitable for this purpose. Several databases are being used (e.g. a cardiogenetic database from the lab as well as a clinical database). It is always a struggle to identify patients on characteristics like mutation, gene, disease etc.'

Table 2 Most common recontacting procedures

Consent given at the first visit where patient agrees to be recontacted $\mathrm{HCP}$ recontacts patient without previous consent

Patient asks HCP to recontact her/him in the future

Follow-up agreed during the first visit

\section{Assessing and recording patient recontacting preferences}

A majority of services (70) indicated that they record patient's recontacting preferences.

Of these, 35 stated that they systematically ask patients about their recontacting preferences. A large majority of these $(31 / 35)$ indicated that this was part of the procedure for obtaining informed consent for genetic testing. The other 35 services responded that they asked patients about their recontacting preferences only occasionally; and a minority of these (14/35) did it as part of the procedure for obtaining informed consent for genetic testing.

A small minority $(5 / 35)$ of the genetic centres that did not record patient recontacting preferences reported that they asked patients about their recontacting preferences on the informed consent form for genetic testing.

The recording of patients' recontacting preferences varied considerably (e.g. some centres recorded patients' preferences only if patients asked not to be recontacted, while others systematically recorded any preference). A minority of the centres that declared they record patients' preferences systematically or occasionally stated that they did not ask that in the consent form. Some explained that they ask about the patients' preference in conversation and record this in the patient file.

'If the patient states that he wants to be recontacted we pay attention and put alerts for it. Most of the time people love to be recontacted. They feel better. They feel that a health professional is caring for them. They feel secure. In our country most of the patients get very happy if they are recontacted by a physician.'

'We get a written consent at the first contact, but we don't ask patients directly whether they wish to be recontacted.'

The majority of the services that said that they did not assess or record their patients' recontacting preferences stated that this was mainly due to a lack of resources available to offer a recontacting service. Some also expressed a concern about raising unrealistic expectations in patients, including fear of potential litigations.

'When you discuss recontact with your patient you are probably raising expectations. At this moment, we are unsure we will be able to deliver on those expectations'

Many services, both those that ask and do not ask about patient preferences, mentioned that they have an 'open-door policy', i.e. HCPs encourage patients to get back in touch 
with the genetics department to check if there are advances and/or to update HCPs about important changes in the family.

Some respondents justified not recording patients' recontacting preferences in their centres by commenting that, in their experience, patients had never or rarely expressed a desire not to be recontacted.

'It rarely happens that a patient asks not to be recontacted, so we do not ask it as a routine. It is more likely that some patients refuse the possibility to continue with further evaluations, when recontacted after many months/years.'

\section{Patient's preferences not to be recontacted}

When asked hypothetically whether there might be reasons for HCPs to recontact patients even if patients indicate they do not want to (or they do not want their relevant family members to be recontacted), 67 genetic centres responded affirmatively. The most common reasons given were the availability of new relevant clinical information for the patient, or for a family member. In a few cases respondents stated that the law of their country requires them to recontact patients in the light of clinically relevant information irrespective of patient wishes, and other respondents reported that they used the primary care physician to facilitate the communication.

\section{'Our Ministry of Health has informed us that we may contact persons in certain conditions for example identification of a clear pathogenic mutation for a disease where treatment and/or prevention are possible,}

Eight of these respondents observed that patients might have changed their mind from the last contact with the clinic/time of consent:

'If extremely important and clinically relevant information becomes available, patients should be notified, as their views might change over time (they might also not be aware of what they are rejecting)'

Other justifications for recontacting patients irrespective of their preferences were the implication of new information for future reproductive risk (for patients or relatives), and possible prenatal diagnosis, and the correction of analytical mistakes.

A summary of the reasons is provided in Table 3 (respondents could give more than one answer).
Among the 38 centres that responded they would always respect patient preferences not to be recontacted, 23 justified their position by referring to the patient's right not to know, and 7 raised legal issues (e.g. the fact that recontacting is forbidden by law in their country).

'Our law generally forbids health care professionals from reaching out to people or their families. However, in unusual circumstances it can be done. If, for example, there was a new therapy that could effectively cure a serious disorder we would consider making an exception to the 'do not contact' rule.'

It is important to note that a considerable number of respondents commented that they had found it difficult to answer this question (irrespective of the answer given), mostly on the basis that they have never or rarely come across the situation of a patient expressing a desire not to be recontacted. This is in line with what was observed above (see section 'Assessing and recording patient recontacting preferences').

\section{HCPs view on the implementation of recontacting}

Fifty-six centres responded that recontacting systems should be implemented. This position was common among many of the centres that stated they recontact routinely using established procedures. Thirty-nine centres were not sure, and ten centres answered negatively.

\section{Arguments in favour of implementing routine recontacting}

The main arguments for implementing routine recontacting systems were the improvement of the knowledge of the clinician, and the desire to provide the best service to the patients and families.

Respondents also commented that recontacting is becoming more important because clinical whole-genome approaches deliver many more genetic variants for

Table 3 Most common reasons for recontacting patients (and relevant family members) even if they indicate they do not want to be recontacted

\begin{tabular}{ll}
\hline & $N$ \\
\hline Relevant clinical information (actionable) for the patient & 44 \\
Relevant information for a family member (including children) & 16 \\
Possibility that patients might change their recontacting & 8 \\
preferences as time lapse & \\
Implication on reproductive risk and possibility of prenatal & 6 \\
diagnosis/PGD & 3 \\
\hline
\end{tabular}


interpretation. Some pointed out that new, relevant information (alongside reinterpretation of VUSs and correction of misleading/outdated information) could change management decisions for patients and offer opportunities for family screening, prevention and new reproductive choices. Moreover, it was argued that recontacting could have a preventative value, reducing the cost of healthcare.

Here are some illustrative quotations of the 'pro' arguments: '[Recontacting offers] the possibility to play out a
preventive medicine'

'Genetics is advancing very fast. Every day we have new information, new techniques, better knowledge to interpret results, an old advice might be misleading.'

'Otherwise [if we do not recontact] there will be unequal conditions for families/patients depending on the time they were tested/counselled.'

However, some respondents expressed concerns about the implementation of recontacting, or the possibility of the development of recontacting guidelines. The main argument was that genetics services are long-established in certain European countries and less established in others and that the implementation of recontacting or the development of guidelines may further increase this disparity.

'It [the implementation of recontacting] would be an ideal scenario. However, we should consider variable level of genetic service development in Europe. If such a system is recommended, it should be recommended only for services that have reached higher standards.'

\section{Arguments against implementing routine recontacting}

The principal arguments against implementing routine recontacting systems were the lack of time, infrastructures, resources and staff to develop systematically recontacting procedures.

Other common arguments against the implementation of routine recontacting systems were related to the patient's right not to know, and concerns about causing anxiety and raising unrealistic expectations in patients, and for the possible legal consequences if such systems established a new standard of care without the additional resources required for it to be a sustainable activity.

A summary of both the arguments in favour and against is reported in Table 4.
Here are some illustrative quotations of the 'against' arguments:

'People may have forgotten they were counselled/ tested and have lived in happy ignorance. They might become worried when confronted with a risk of developing a serious disease. They may think about relatives who died at a young age due to a genetic disease [for] which treatment/prevention are now possible/available.'

'If you recontact regularly, this could raise unrealistic hopes that there would be better diagnosis and treatment.'

Some respondents pointed out that HCPs working in 'mainstream' specialties, for instance paediatricians and cardiologists, are supposed to have a more ongoing relationship with patients compared to the clinical geneticists. 'Mainstream' specialists, as well as primary care physicians, were seen by these respondents as having potentially more responsibility for recontacting patients.

\section{Discussion}

\section{The problem of defining recontacting}

The survey answers revealed a multiplicity of understandings of the term 'recontacting'.

In the introduction to our survey, as well as in the covering letter sent to the genetics centres with the link to the survey, we defined recontacting as contacting patients who have previously been discharged from a genetic service. Yet, some appeared to conflate routine follow-up programme or even the post-test result counselling with recontacting. Some respondents referred to recontacting as being a process exclusively initiated by the clinician, others as something that can be initiated by either clinician or patient, while others suggested it could be initiated by the patient (or support groups) alone.

It is important to reach an 'operational definition' that can be useful to clinical genetics centres, HCPs, and laboratories in different countries. It is also important to highlight that it can be particularly challenging for mainstream specialties to differentiate recontacting from follow-up. Moreover, mainstream specialties may not receive up-to-date information on DNA variant interpretation, as this happens predominantly in the genetics centres, or they may fail to contact the laboratory or the referring geneticist if new genetic information becomes available. 
Table 4 Main arguments in favour and against the implementation of recontacting systems

Arguments in favour of the implementation of routine

recontacting systems

Better knowledge, diagnosis and care

Make the recontact easier

Facilitate follow-up and strengthen trust between patient

- caregiver

It is HCP's responsibility towards the patients

Avoid unequal conditions for families/patients depending on the

time they were tested/counselled.

Prevent recurrence of genetic diseases in the family

Arguments against the implementation of routine recontacting systems

Lack of time, resources and staff to put it into practice

Respecting patients and families' preferences and right not to know

Lack of knowledge on the current clinical status of the patient and on whether recontacting could be

emotionally acceptable (anxiety)

Legal issues related to the duty for recontact

None

Patients' data and address might change over time and they could be lost

As stated by one respondent: "Not all the genetic tests are requested by a clinical genetics department. As we mainstream more tests this work will be undertaken by nongeneticists and therefore the patients being tested will not be under our care".

\section{Recontacting occurs in Europe, but not systematically}

Recontacting appeared to be important to respondents, even if only a minority of European centres routinely recontact. Many respondents who affirmed that they recontacted only occasionally highlighted that they would and should recontact more systematically, supporting the idea that recontacting can promote a better quality of care in clinical practice.

A significant number of respondents tended to ask patients if they would like to be recontacted (and record patient preferences). However, a majority of clinicians would hypothetically recontact patients or family members even if the patients asked not to be recontacted if this new information was clinically relevant. This response appears to be in line with the ESHG recommendations on the use of whole-genome sequencing in healthcare: "Patients' claims to a right not to know do not automatically over-ride professional responsibilities when the patient's own health or that of his or her close relatives is at stake" [22], p. 583. Even if current regulation of individual countries specifically recognises an individual's right not to know diagnostic (genetic) information, in the majority of countries there are no rules that define the circumstances in which the clinicians should go against this right $[7,23]$.
Without a professional consensus about whether and in what circumstances patients should be recontacted, the decision whether or not to recontact the patients weighs on HCPs [4].

Moreover, in some countries there may also be a tension between the law and the clinical ethics.

\section{Different recontacting procedures}

Recontacting procedures varied between different centres and countries. Most involved the use of databases for recontacting purposes, but there was a considerable diversity in practices regarding the use of clinical genetics databases. This may be the result of historical and resource allocation differences.

Some centres that stated they do not recontact patients, reported asking about patients' recontacting preferences in the consent form. This suggest that these centres may be willing, but not able, to recontactdue to lack of staffing and resources; therefore, they currently limit themselves to only recording patients' preferences.

Barriers to the development or implementation of recontacting procedures included lack of resources, concerns about potential negative psychological consequences of recontacting, unclear operational definitions of recontacting, policies that prevent HCPs from recontacting and difficulties in locating patients after their last contact. Any future recommendation about recontacting needs to consider these barriers. 


\section{Implementation of recontacting systems}

Fifty-six centres answered that recontacting systems should be implemented.

Among the reasons not to do so, the main issue was the lack of time, resources and staff. This survey has also highlighted the different levels of development of European genetics centres in terms of procedures related to recontacting and the availability of staff/resources to recontact. This finding can in turn be explained by the fact that the development (and establishment) of the specialties of medical genetics and genetic counselling is highly variable across different European countries. In some countries, other professionals, medical and non-medical, often without an adequate training in genetics, evaluate and provide counselling to the patients. Furthermore, most clinical genetics services are multi-disciplinary. This means that any recontacting recommendations should not be prescriptive to a specific HCP group, but it is the organisational policy of each country as to which speciality should define the recontact process. However, it is also important that any recontacting recommendation contributes to a harmonisation of service standards rather than an amplification of discrepancies and service inequities within and between countries.

The concern of recontacting patients inappropriately without respecting their autonomy and self-determination was one of the major factors weighing against the implementation of recontacting, alongside with a concern for the psychological consequences of recontacting.

As suggested by Carrieri et al. [24], a first step to mitigate these risks could be for HCPs to discuss with patients-routinely as part of the consent process for genetic testing or whenever patient data are collected and recorded-the possibility of recontact, and assess patients expectations and preferences. Such discussion between HCP and patient would allow to clarify roles and expectations, and to minimise the risk of giving patients 'false hope' or 'false security'.

The ESHG Guidelines for diagnostic next-generation sequencing state: "whenever major changes are made to the test, quality parameters have to be checked, and samples have to be re-run. The laboratory should define beforehand what kind of samples and the number of cases that have to be assayed whenever the method is updated or upgraded" [23]. Well-established procedures, such as a database shared between laboratories and clinicians, or systems to send updates from the laboratory to clinicians (realities already reported by some of our respondents) could help to trigger recontacting in those centres (the majority of the European genetic centres) that still do not benefit from a routine feedback from the laboratory, as suggested by some of the survey's respondents. A question to consider now is whether the preventative value of reanalysing, reinterpretation and recontacting in practice reduce the overall cost of healthcare.

\section{Limitations of the study}

It has been problematic to identify all clinical genetic services across Europe. This is also due to a lack of policies in Europe that establish standards, and requirements necessary to define a clinical genetic centre.

When it was possible to determine the response rate of the centres in answering the survey, this varied considerably among the different countries.

Possibly because they did not want to be judged on the basis of their responses to the survey, some respondents did not report from which clinical centre they were writing and they only put the name of their country.

It is also important to point out that the UK survey was administered between October 2014 and July 2015, around 2 years before the administration to the other European countries. Although we think the results are comparable, we also recognise that in the 2-year period between the administrations of the surveys, clinical genetics technologies and procedures may have changed in ways that may be significant to recontacting, both in the UK and in the other European countries.

Acknowledgements We are indebted to Professor Milan Macek, chair of the EU National Human Genetics Societies, the Presidents and the boards of the National Human Genetics Societies of European countries and all the Clinical Genetics Departments and Units who collaborated and participated in our survey. This work would not have been possible without them.

\section{Compliance with ethical standards}

Conflict of interest The authors declare that they have no conflict of interest.

\section{References}

1. Fitzpatrick JL, Han C, Costa T, Huggins MJ. The duty to recontact: attitudes of service providers. Am J Hum Genet. 1999;64:852-60.

2. Hirschhorn K, Fleisher LD, Godmilow L, Howell RR, Lebel RR, McCabe ERB. Duty to re-contact. Policy statement: Social Ethical and Legal Issues Committee of the American College of Medical Geneticists. Genet Med. 1999;1:171-2.

3. Hunter AGW, Sharpe N, Mullen M, Meschino WS. Ethical, legal, and practical concerns about recontacting patients to inform them of new information. Am J Med Genet. 2001;103:265-76.

4. Carrieri D, Dheensa S, Doheny S, et al. Recontacting in clinical practice: an investigation of the views of healthcare professionals and clinical scientists in the United Kingdom. Eur J Hum Genet. 2017;25:275-9.

5. Otten E, Plantinga M, Birnie E, et al. Is there a duty to recontact in light of new genetic technologies? A systematic review of the literature. Genet Med. 2015;17:668-78.

6. Carrieri D, Dheensa S, Doheny S, et al. Recontacting in clinical practice: the views and expectations of patients in the United Kingdom. Eur J Hum Genet. 2017;25:1106-12.

7. Laurie G. Privacy and the right not to know: a plea for conceptual clarity./ Laurie, Graeme. The right to know and the right not to 
know: genetic privacy and responsibility. In: Ruth Chadwick; Mairi Levitt; Darren Shickle. 2nd. ed. Cambridge University Press. 2014; p. 38-52.

8. Sexton AC, Sahhar M, Thorburn DR, Metcalfe SA. Impact of a genetic diagnosis of a mitochondrial disorder 5-17 years after the death of an affected child. J Genet Couns. 2008;17:261-73.

9. Bernard LE, McGillivray B, Allen MIV, Friedman JM, Langlois S. Duty to re-contact: a study of families at risk for Fragile X. J Genet Couns. 1999;8:3-15.

10. Pyeritz RE. The coming explosion in genetic testing-is there a duty to recontact? N Engl J Med. 2011;365:1367-9.

11. Murray ML, Cerrato F, Bennett RL, Jarvik GP. Follow-up of carriers of BRCA1 and BRCA2 variants of unknown significance: variant reclassification and surgical decisions. Genet Med. 2011;13:998-1005.

12. Knoppers BM. Duty to recontact: a legal harbinger? Am J Med Genet. 2001;103:277-277.

13. Dean JCS, Fitzpatrick DR, Farndon PA. Genetic register in clinical practice: a survey of UK clinical geneticists. J Med Genet. 2000;37:636-40.

14. Rantanen E, Hietala M, Kristoffersson U, Nippert I. Regulations and practices of genetic counselling in 38 European countries: the perspective of national representative. Eur $\mathrm{J}$ Hum Genet. 2008;16:1208-16.

15. Shirts BH, Parker LS. Changing interpretations, stable genes: responsibilities of patients, professionals, and policy makers in the clinical interpretation of complex genetic information. Genet Med. 2008; 10:778-83.

16. Burton H, Alberg C, Stewart A. Mainstreaming genetics: a comparative review of clinical services for inherited cardiovascular conditions in the UK. Public Health Genom. 2010;13: 235-45.

17. McAllister M, Payne K, MacLeod R, Nicholls S. What process attributes of clinical genetics services could maximise patient benefits? Eur J Hum Genet. 2008;16:1467-76.

18. Kausmeyer DT, Lengerich EJ, Kluhsman BC, Morrone D, Harper GR, Baker MJ. A survey of patients' experiences with the cancer genetic counseling process: recommendations for cancer genetics programs. J Genet Couns. 2006;15:409-31.

19. Hart JT. The inverse care law. Lancet. 1971;297:405-12.

20. Carrieri D, Lucassen AM, Clarke AJ, et al. Recontact in clinical practice: a survey of clinical genetics services in the United Kingdom. Genet Med. 2016;18:876-81.

21. Bauer MW. Classical content analysis: a review. In: M. W. Bauer \& G. Gaskell (Ed.). Qualitative researching with text, image and sound; 2000 London, UK: Sage p. 131-51.

22. van El,CG, Cornel MC, Borry P, et al. Whole-genome sequencing in health care. Eur J Hum Genet. 2013;21:S1-S5.

23. Berkman BE. Refuting the right not to know. J Health Care L Pol'y. 2016;19:1.

24. Carrieri D, Dheensa S, Doheny S, et al. Recontacting in clinical genetics and genomic medicine? We need to talk about it. Eur J Hum Genet. 2017;25:520-1.

\section{Affiliations}

Fabio Sirchia ${ }^{1}$ - Daniele Carrieri $\mathbb{D}^{2}$ - Sandi Dheensa ${ }^{3}$ Caroline Benjamin ${ }^{4,5} \cdot$ Hülya Kayserili ${ }^{6}$. Christophe Cordier $\mathbb{D}^{7}$. Carla G. van $\mathrm{El}^{8}$ - Peter D. Turnpenny ${ }^{9} \cdot$ Bela Melegh $^{10}$ - Álvaro Mendes ${ }^{11}$. Tanya F. Halbersma-Konings ${ }^{12} \cdot$ Irene M. van Langen $^{12} \cdot$ Anneke M. Lucassen $^{3,13}$ - Angus J. Clarke $\mathbb{I}^{14}$. Francesca Forzano ${ }^{15}$. Susan E. Kelly ${ }^{2}$

1 Department of Medical Sciences and Medical Genetics Unit, Città della Salute e della Scienza University Hospital, University of Torino, Torino, Italy

2 Egenis, University of Exeter, Exeter, UK

3 Clinical Ethics and Law, Faculty of Medicine, University of Southampton, Southampton, UK

4 School of Community Health \& Midwifery, University of Central Lancashire (UCLan), Preston, England, UK

5 Liverpool Women's NHS Hospital Trust, England, UK

6 Department of Medical Genetics, Koç University School of Medicine İstanbul, İstanbul, Turkey

7 Department of Genetics, SYNLAB Lausanne, Lausanne, Switzerland

8 Department of Clinical Genetics, Section Community Genetics and Amsterdam Public Health Research Institute, VU University Medical Center, Amsterdam, The Netherlands
9 Clinical Genetics, Royal Devon \& Exeter NHS Foundation Trust, Exeter, UK

10 Department of Medical Genetics, and Szentagothai Research Ctr, University of Pécs Medical School, Pécs, Hungary

11 UnIGENe and CGPP-Centre for Predictive and Preventive Genetics, IBMC - Institute for Molecular and Cell Biology, i3SInstituto de Investigação e Inovação em Saúde, Universidade do Porto, Porto, Portugal

12 Deparment of Genetics, University Medical Centre Groningen, University of Groningen, Groningen, The Netherlands

13 Wessex Clinical Genetics Service, University Hospitals Southampton NHS Foundation Trust, Southampton, UK

14 School of Medicine, Cardiff University, Cardiff, UK

15 Clinical Genetics Department, Guy's Hospital Guy's \& St Thomas' NHS Foundation Trust London UK 